\title{
USE OF RESULTS FROM HONING TEST MACHINES TO DETERMINE ROUGHNESS IN INDUSTRIAL HONING MACHINES
}

Irene Buj-Corral1,* Lourdes Rodero-De-Lamo2, and Lluís Marco-Almagro2

1 Department of Mechanical Engineering, School of Industrial Engineering of

Barcelona, Universitat Politècnica de Catalunya, Av. Diagonal, 647, 08028 Barcelona, Spain

2 Department of Statistics and Operations Research. School of Engineering of Barcelona, Universitat Politècnica de Catalunya, Av. Diagonal, 647, 08028 Barcelona, Spain

*Corresponding author. Tel. +34934054016. Fax. +34934016693.

E-mail address of corresponding author: irene.buj@upc.edu (I. Buj-Corral)

Other e-mail addresses: lourdes.rodero@upc.edu (L.Rodero-De-Lamo);

lluis.marco@upc.edu (Ll. Marco-Almagro). 


\begin{abstract}
In the present work, a new methodology is presented to translate roughness results from a test machine to different industrial machines without the need to stop production for a long time. First, mathematical models were searched for average roughness Ra in finish honing processes, in both a test and an industrial machine. Regression analysis was employed for obtaining quadratic models. Main factor influencing average roughness Ra was grain size, followed by pressure. Afterwards, several experiments were simulated in the common range of variables for the two machines using the models for roughness. A new variable DifRa corresponding to the difference between roughness values from the test machine and the industrial machine was defined and a quadratic model was obtained. Once DifRa is modeled, it is possible to predict roughness in a different industrial honing machine from results of the test machine by performing a few experiments in the industrial machine and translating the curves. This will reduce the number of tests to be performed in industrial machines. The suggested new methodology has been tested with two more roughness parameters, $\mathrm{Rz}$ and Rk, proving its validity.
\end{abstract}

Keywords: Honing, roughness, regression analysis.

\title{
1 Introduction
}

In interior honing processes, a honing head provided with abrasive stones is used for removing material from the internal surface of cylinders. Honing is employed to improve shape, dimensional precision and surface finish of previously machined parts. Usually, the honing head combines a linear alternate movement with rotation, so that abrasive grains provide a crosshatch pattern on the workpieces' surface [1]. Main 
advantages of interior honing are high material removal rate up to $0.6 \mathrm{~mm} / \mathrm{min}$, much higher than that obtained in lapping processes, with typical values of $0.5 \mu \mathrm{m} / \mathrm{min}$, the fact that a lower pressure is usually employed than in grinding processes, making it possible to control roundness and dimensions of the part, and improved surface texture with cross-hatch pattern [1,2] . Main drawbacks are that it is a low-velocity process, it does not allow correction of misalignment and that it has a reduced area of application [1]. Surface finish obtained by means of honing is related to friction between piston and cylinder liners [3] as well as to tool wear [4].

Honing is a multistage process that usually comprises rough, semifinish and finish operations. Each successive operation is performed with finer grain size of abrasive in order to achieve a smoother surface. In industry, both vertical and horizontal configurations are used. In order to improve productivity, three-head vertical machines that allow honing with three cylinders working simultaneously, in three different stages, can be used.

In the literature, few authors have obtained mathematical models for roughness in honing processes. Troglio carried out a full three-level design of experiments, with factors like abrasive grain size, lubricating oil and workpiece material, and responses such as roughness Ra or Rk family (Abbott-Firestone) parameters. In addition, he studied influence of the process on roundness and cylindricity. He also studied influence of tool wear on consumed power, material removal rate and specific energy [5]. Bai and Zhang studied how to increase efficiency in honing, with variables such as pressure, speed and cross-hatch angle. Best material removal rate was observed at a cross-hatch 
angle between $40^{\circ}$ and $60^{\circ}$. Pressure was one of the most influential parameters on roughness [6]. Kanthababu et al employed rotation speed, linear speed, pressure, honing time and plateau-honing time as variables in a three-level design of experiments. Responses were roughness parameters of the Abbott-Firestone curve (Rk, Rpk, Rvk, Mr1, Mr2) [7]. Main factors affecting surface roughness were pressure and honing time. In plateau honing processes, Pawlus, Cieslak and Mathia employed diamond abrasive stones for honing cast iron cylinders, with grain size 151 and 76 (FEPA) in rough and semifinishing steps, which correspond to size ranges 150/125 and 75/63 $\mu \mathrm{m}$ respectively, and 15 in plateau honing, which corresponds to size range 15/25 $\mu \mathrm{m}$ [8]. They considered working pressure in honing and in plateau-honing, as well as plateauhoning time. They searched a correlation between roughness parameters related to the Abbott-Firestone curve and roughness parameters related to the probability curve, both in two and three dimensions. They found that probability parameters are independent, while Abbott-Firestone parameters show linear dependence. Buj-Corral et al. obtained models for average roughness Ra as a function of both machine parameters (pressure, linear speed and tangential or rotation speed) and honing stone parameters (grain size, density of abrasive) in rough honing. They found that, in the range studied, grain size of abrasive and pressure are main variables affecting roughness and material removal rate [9]. Lawrence and Ramamoorthy studied rough, finish and plateau honing processes by means of robust process design and gray-relational analysis in order to optimize Rk family parameters, Rz and honing angle [10]. Vrac et al. found models for average roughness in honing processes with diamond abrasive stones. Main factor influencing roughness was cutting speed for grain size 181 (size range 180/150 $\mu \mathrm{m}$ ) and specific pressure of finishing honing for grain size 151 (size range 150/125 $\mu \mathrm{m}$ ) [11]. It is well 
known that the higher grain size, the higher surface roughness is [12]. In addition, pressure increases roughness, while tangential speed reduces it [13].

In a previous paper by the authors of the present paper, average roughness Ra obtained in a test machine and in an industrial machine in semifinish honing processes were compared [14]. In the present paper, parameters Ra, Rz and Rk were compared for both a test and an industrial honing machine in finish processes. Mathematical models were obtained for roughness parameters as a function of five process variables. For the three roughness parameters studied it was observed that shapes of models for test machine and for industrial machine are similar, although they are separated by a certain distance. For this reason, the difference between values from the two machines was modelled. Assuming that different industrial honing machines will have a similar behavior regarding roughness, the models for differences will allow predicting roughness values to be obtained in a certain industrial machine by only performing few tests and translating values from the previously obtained models of the test machine, without having to stop production a long time in order to perform time-consuming tests. Once the method had been tested for three different roughness parameters, Ra, Rz and Rk, a general methodology was defined. It will allow determining roughness in industrial machines from roughness in test machines. The results guarantee the validity of the methodology.

\section{Materials and methods}

\subsection{Honing tests}


Two different machines were employed: a horizontal test machine (Figure 1) and a vertical Honingtec industrial machine (Figure 2). Steel St-52 cylinders of $80 \mathrm{~mm}$ internal diameter were machined. Length of cylinders was $100 \mathrm{~mm}$ in the test machine and $390 \mathrm{~mm}$ in the industrial machine. Finish honing tests were carried out.

Characteristics of performed tests are presented in Section 2.2.

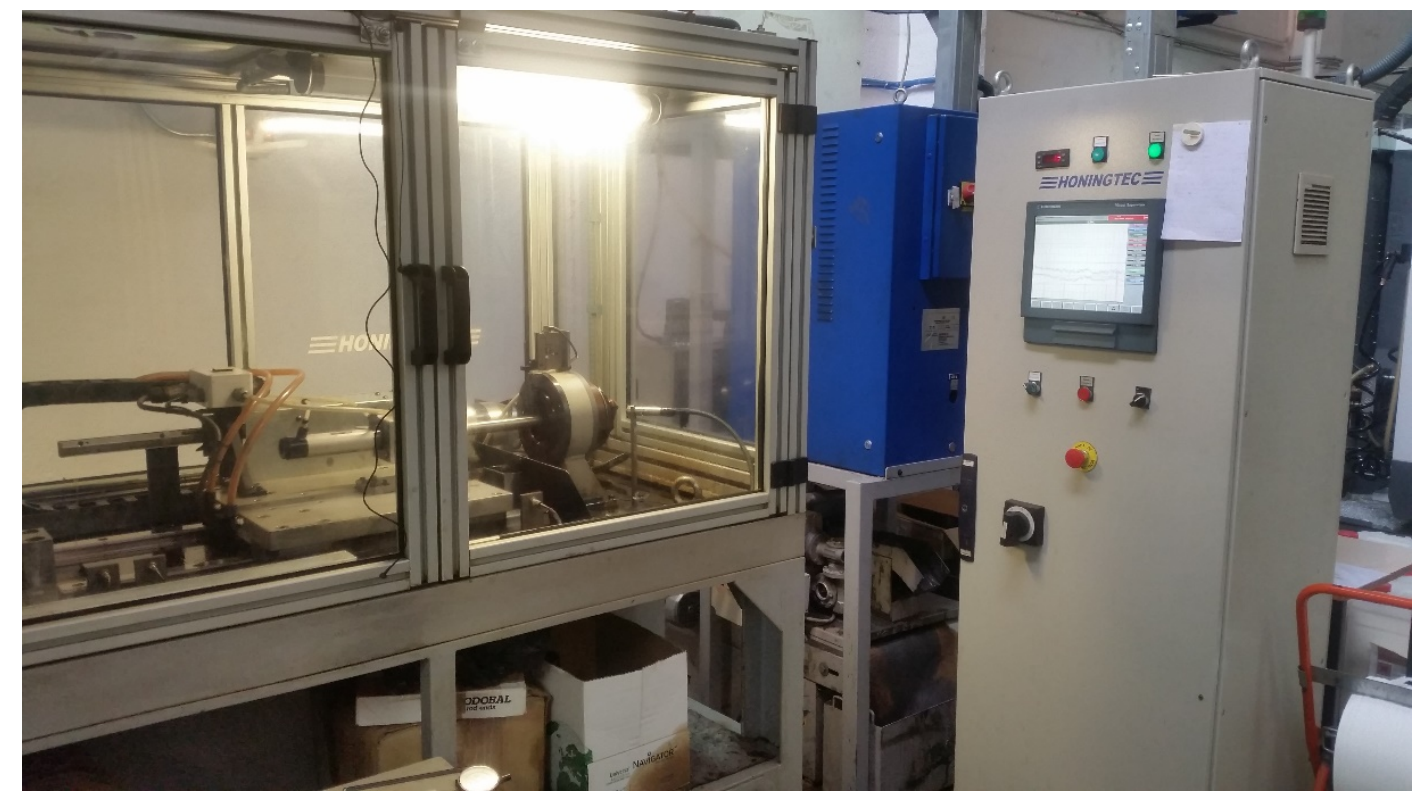

Figure 1. Test honing machine 


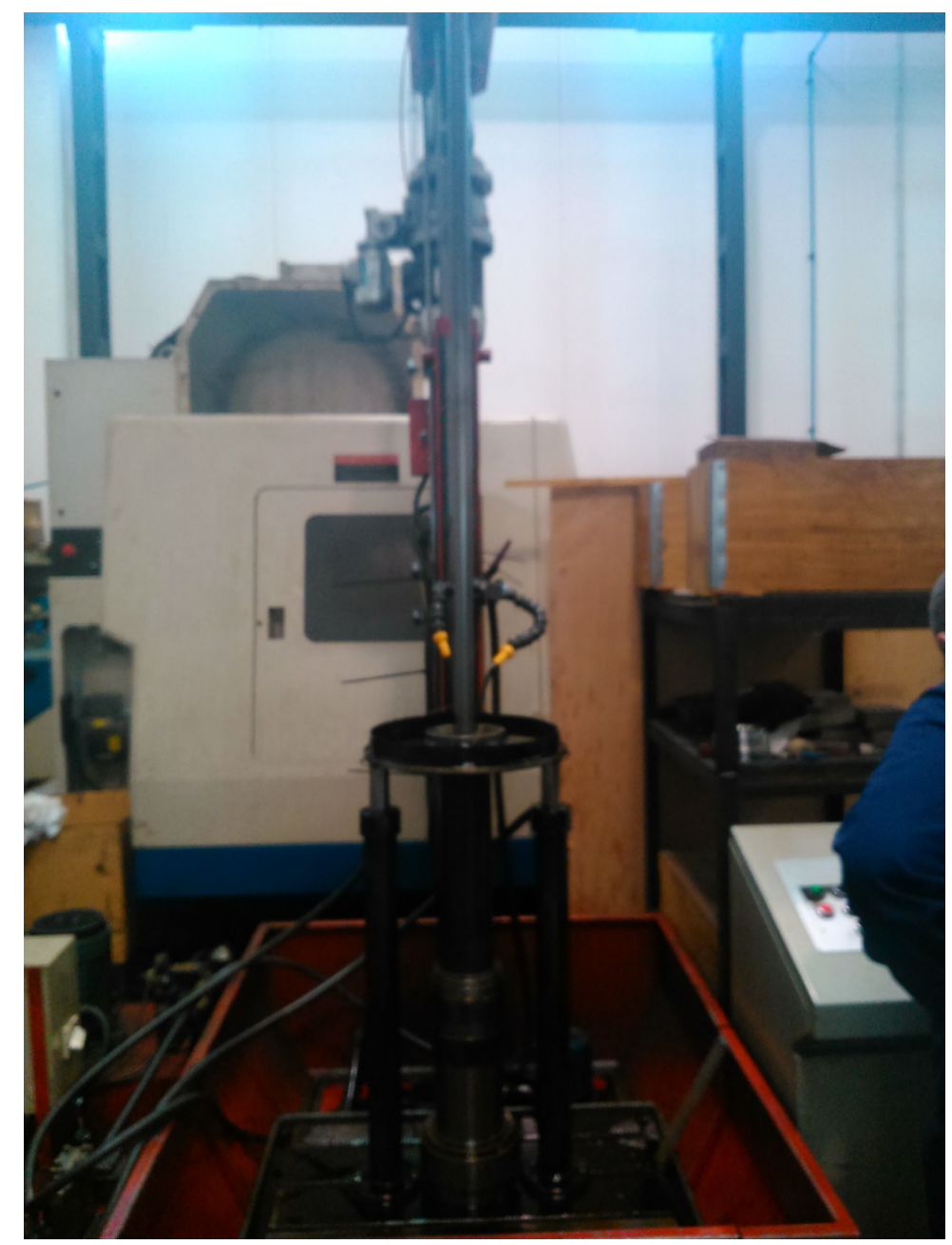

Figure 2. Industrial honing machine

A Hommel W5 roughness meter was employed for measuring roughness. For each cylinder, 9 points were measured along a diametric circumference in the internal surface of cylinders [15]. Measurements were performed at the mid-height center of the cylinders in order to avoid ends where honing head decelerates and accelerates. It corresponds to $50 \mathrm{~mm}$ from both ends for the test machine and to $195 \mathrm{~mm}$ from both ends for the industrial machine.

\subsection{Statistical tools}


In order to obtain quadratic mathematical models for roughness, experiments according to a central composite design were performed. A central composite design has three kinds of experimental data points: cube, axial and central points. A graphical representation of the structure of this type of experiments is shown in Figure 3 (for the situation with only 3 factors) [16]. Cube points are runs coming from a two-level factorial design (either full or fractional). Central points are runs performed at the middle value of all the variables. These central points are replicated, thus allowing an estimation of the variance of the response, and the performance of a lack of fit test. Finally, the axial points are located at a certain distance (called $\alpha$ ) from the center of the cube. These axial points allow the estimation of quadratic terms in the model, if needed.

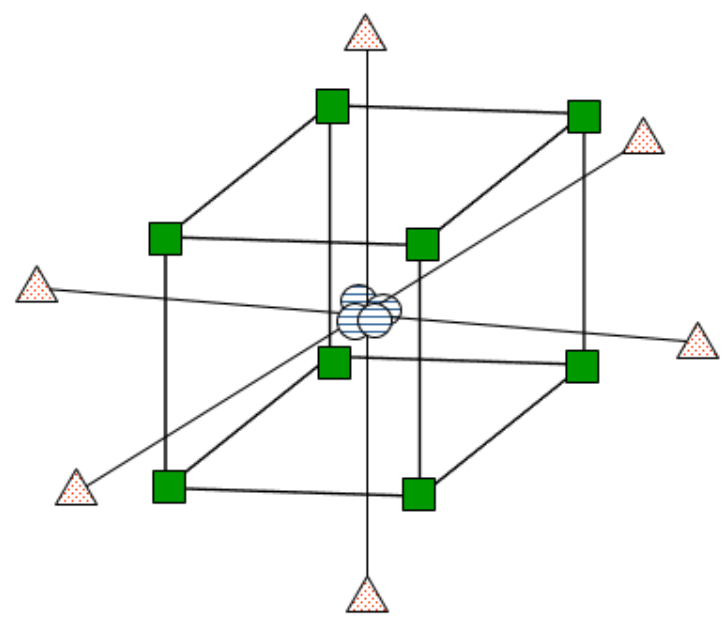

\section{Central experiments}

Cube experiments

Axial experiments

Figure 3. Representation of a central composite design when having 3 factors

In the present paper, a $2^{5-1}$ factorial design was designed for the five factors [12]: grain size Gs [17], abrasive density De [18], pressure $\operatorname{Pr}\left(\mathrm{N} / \mathrm{cm}^{2}\right)$, tangential speed Vt $(\mathrm{m} / \mathrm{min})$ and linear speed $\mathrm{Vl}(\mathrm{m} / \mathrm{min})$. Gs corresponds to grit range in micrometer, while De is related to weight of abrasive in carats for a certain abrasive stone volume in cubic centimeters. 
Face centered axial points were added (thus having an $\alpha=1$ ), as well as 6 central points. Two replicates were considered with a total amount of 64 runs. This experiment design allows the estimation of all effects up to order two (linear effects, order two interactions, and full quadratic effects). Appropriateness and sufficiency of this second order function for representing the influence of the five factors on roughness (Ra) was assessed using a residuals validation and the lack of fit test.

The proportion of variability of the roughness explained by the five factors is measured with the coefficient of determination of the regression $\left(R^{2}\right)$ and the adjusted $R^{2}$. The adjusted $R^{2}$ explains the percentage of response variability explained by the model, adjusted for the number of predictors. This adjustment takes into account the spurious increase of $R^{2}$ when a new regressor is added to the model. In this sense, the adjusted $R^{2}$ only increases if the added term is significant and really improves the model. Equation 1 shows the formula for computing the adjusted $R^{2}$ based on the $R^{2}$ of the regression equation, the number of predictors $p$ and the number of data points $N$.

$$
R_{\text {adjusted }}^{2}=1-\frac{\left(1-R^{2}\right)(N-1)}{N-p-1}
$$

The higher the adjusted $R^{2}$, the better the model can explain the response. Thus the adjusted $R^{2}$ will be used to assess the quality of the obtained model. 
Table 1 contains the values of the variables employed in the finish honing experiments conducted. Levels for linear and tangential speed are not the same for both the test and the industrial machine, because of differences in the configuration of both machines.

Table 1. Ranges of variables employed

\begin{tabular}{|c|c|c|}
\hline Variable & $\begin{array}{c}\text { Test } \\
\text { machine }\end{array}$ & $\begin{array}{c}\text { Industrial } \\
\text { machine }\end{array}$ \\
\hline Gs (FEPA) & $15-30$ & $15-30$ \\
\hline De (ISO 6104) & $10-20$ & $10-20$ \\
\hline $\mathrm{Pr}\left(\mathrm{N} / \mathrm{cm}^{2}\right)$ & $400-700$ & $400-700$ \\
\hline $\mathrm{Vt}(\mathrm{m} / \mathrm{min})$ & $30-50$ & $15-35$ \\
\hline $\mathrm{Vl}(\mathrm{m} / \mathrm{min})$ & $20-40$ & $15-25$ \\
\hline
\end{tabular}

Once data from the experiments were obtained, regression analysis was carried out to find models that explain the response in both machines (test machine and industrial machine), in terms of the five studied variables. The models were validated using residuals analysis and lack of fit tests in order to obtain the best possible model for the available data. Minitab 17 statistical software [19] was employed for the analysis.

In order to quantify the importance of each variable (Gs, De, Pr, Vt and Vl) in explaining the response, a relative importance index was computed for each factor in the model [20]. The relative importance index (RII) for factor $x_{i}$ is computed using the expression in Equation 2:

$$
R I I=\frac{R_{y\left(x_{1}, \cdots, x_{p}\right)}^{2}-R_{y\left(x_{1}, \cdots, x_{i-1}, x_{i+1}, \ldots, x_{p}\right)}^{2}}{R_{y\left(x_{1}, \cdots, x_{p}\right)}^{2}} \cdot 100
$$


Where $R_{y\left(x_{1}, \ldots, x_{p}\right)}^{2}$ is the coefficient of determination of the full model and $R_{y\left(x_{1}, \ldots, x_{i-1}, x_{i+1}, \ldots, x_{p}\right)}^{2}$ is the coefficient of determination of the model where all terms having $x_{i}$ have been removed. This difference, divided by a normalizing factor and expressed as a percentage, shows the relative importance of factor $x_{i}$. Once the RII for each factor is computed, results are rescaled so that the total sum is $100 \%$.

Based on the study of the relative importance of factors in the models, it is possible to simplify them as much as possible into reduced models that offer only a slight decrease in the explanation of the response, but that are much simpler to be interpreted.

Once the models for both test and industrial machines are obtained, one can use them to generate predicted values of the response in given experimental conditions. This is especially relevant in those situations - as the one in this study - where the experimental ranges of some variables are not the same for the test and for the industrial machine.

The basis for translating results from the test machine to an industrial machine is being able to model the difference in Ra values between both machines. A new variable DifRa was obtained for modelling the distance between simulated roughness values in test and industrial machine, in the following manner (Equation 3):

$$
\text { DifR } a=R a_{\text {test machine }}-R a_{\text {industrial machine }}
$$




\section{Results and discussion}

The obtained experimental were first used to find models for roughness $(\mathrm{Ra})$ for the test and industrial machines. After that, differences between values from both machines (DifRa) was modeled. This model was then used to translate results from the test machine to the industrial machine.

\subsection{Models for roughness ( $\mathrm{Ra})$ in test and industrial machines}

The best possible model using $\mathrm{Ra}$ as response for both the test machine and the industrial machine was computed using a least squares regression procedure. The model for the test machine is presented in Equation 4:

$$
\begin{aligned}
& R a=1.324-0.10188 G s+0.0137 D e-0.0010 P r-0.00698 V t-0.00798 V l+0.002585 G s^{2} \\
& -0.001108 D e^{2}+0.00004 G s \cdot P R-0.000132 G s \cdot V l+0.000033 D e \cdot P r \\
& +0.000249 V t \cdot V l
\end{aligned}
$$

(Eq. 4)

The model has a coefficient of determination $R^{2}=99.07 \%$ and an adjusted $R^{2}=$ $98,87 \%$.

The model for the industrial machine is shown in Equation 5:

$$
\begin{array}{r}
R a=1.164-0.07211 G s+0.01334 D e-0.00773 P r-0.01099 V t-0.00007 V l+0.001133 G s^{2} \\
+0.000627 G s \cdot D e+0.000036 G s \cdot P r+0.00015 G s \cdot V t+0.000013 P r \cdot V t
\end{array}
$$

(Eq. 5)

The model has a coefficient of determination $R^{2}=92.79 \%$ and an adjusted $R^{2}=$ $91,38 \%$. 
Both models are hierarchical models that only keep significant effects, with a level of significance of 0.05 . The models were validated by means of analysis of residuals, which show no dependence, no heteroscedasticity and no departure from normality.

As Figure 4 shows, most important variable affecting roughness in both the test and the industrial machine is Gs, with Pr being on a distant second place. Grain size of abrasive (Gs) influences roughness because the higher grain size, the wider machining marks are. This leads to different surface topographies with different roughness values. Pressure (Pr) also influences roughness in honing processes, since at high pressure grains will leave a deeper mark on the workpiece’s surface, changing surface topography and roughness.

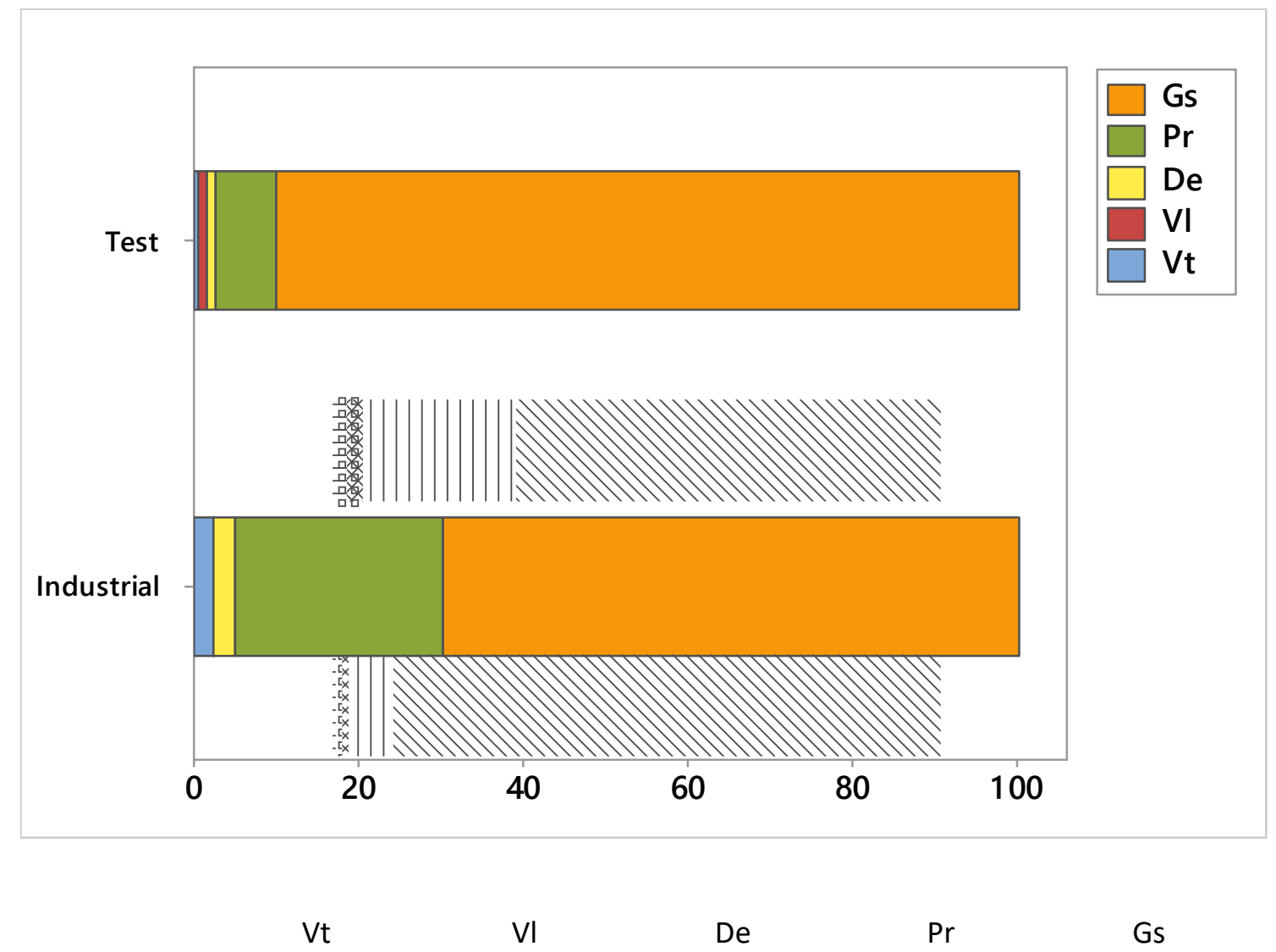




\begin{tabular}{|l|c|c|c|c|c|}
\hline Test & $0.74 \%$ & $0.95 \%$ & $0.96 \%$ & $7.36 \%$ & $90 \%$ \\
\hline Industrial & $2.49 \%$ & $0 \%$ & $2.65 \%$ & $25.11 \%$ & $69.75 \%$ \\
\hline
\end{tabular}

Figure 4. Relative importance of factors for the test and the industrial machines

Although the models for the test and the industrial machines do not have exactly the same significant terms, the response surfaces obtained are in fact very similar in shape (the level of the response varies, being less in the industrial machine), as Figure 5 shows. These response surfaces were obtained for variables Gs and $\operatorname{Pr}$ (most important ones in this process). All other variables were kept constant at their middle values.
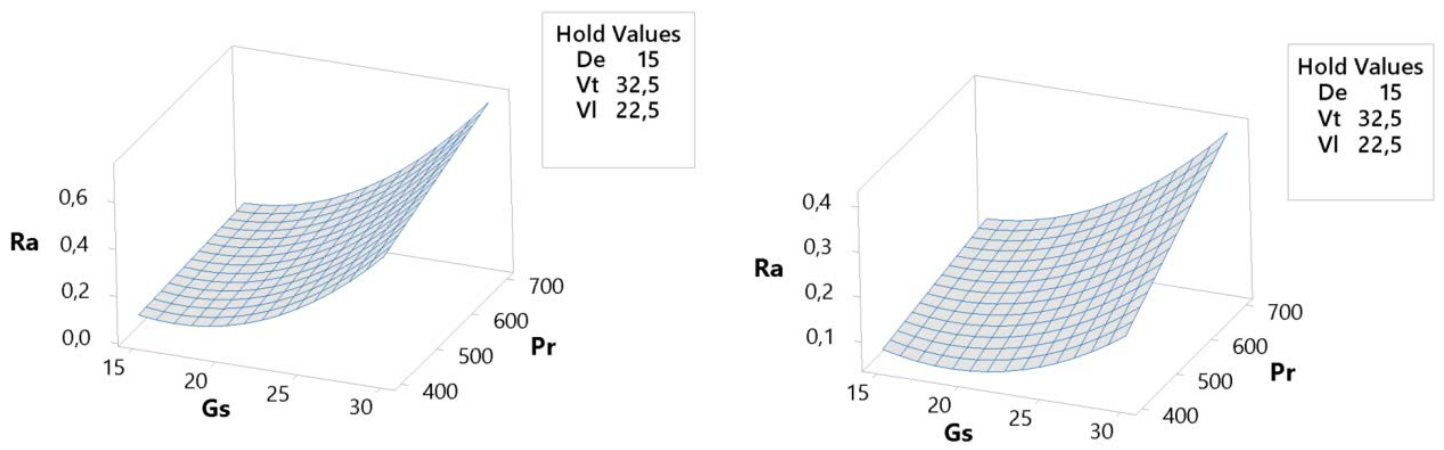

Figure 5. Response surface for Ra in the test machine (left) and the industrial machine (right), for variables Gs and Pr, using the complete models.

Taking into account the fact that only Gs and Pr are important factors when modeling the response surface of $\mathrm{Ra}$, it is possible to create reduced models for both machines. These models will only include linear and - if needed - quadratic terms for Gs and Pr. The reduced model for the test machine is presented in Equation 6: 


$$
R a=0.361-0.0652 G s+0.000386 P r+0.002172 G s^{2}
$$

(Eq. 6)

This model has a coefficient of determination $R^{2}=95.05 \%$ and an adjusted $R^{2}=$ $94,80 \%$.

The reduced model for the industrial machine is provided in Equation 7:

$$
R a=0.346-0.0390 G s+0.000363 P r+0.001133 G s^{2}
$$

(Eq. 7)

This model has a coefficient of determination $R^{2}=79.97 \%$ and an adjusted $R^{2}=$ $77,88 \%$.

Although these reduced models are not the best possible ones, as an analysis of residuals makes evident, the amount of response variability explained is not greatly reduced (the adjusted coefficient of determination $\mathrm{R}^{2}$ decreases, but not in a big amount). Furthermore, response surfaces using the complete or the reduced models are analogous, as a visual comparison between Figures 5 and 6 shows.
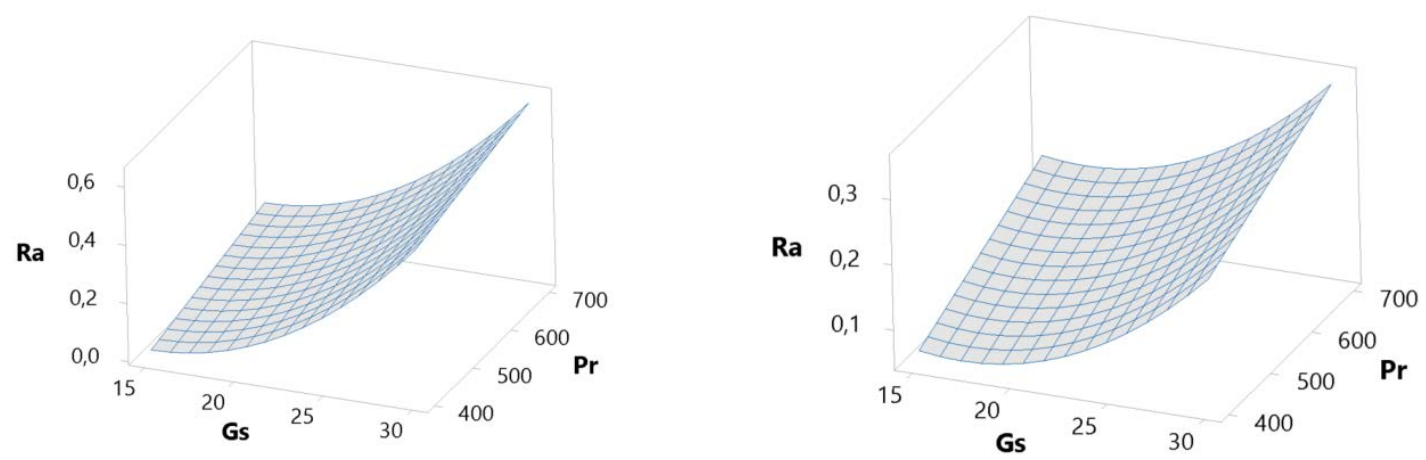

Figure 6. Response surface for Ra in the test machine (left) and the industrial machine (right), for variables Gs and Pr, using the reduced models 
Consequently, it is reasonable to use the reduced models to explain roughness in both the test and the industrial machines. Having these reduced models makes comparison between both machines more feasible.

\subsection{Translation model for $\mathrm{Ra}$}

The purpose of this section is developing a new methodology to predict roughness behavior in the industrial machine based on the model from the test machine. This will be possible due to the fact that the surface responses in the test and the industrial machines are similar, as shown in Section 3.1. Some experiments have to be conducted in the industrial machine to determine in which way it is possible to transfer the test machine model to the industrial machine model. But, hopefully, only very few experiments in the industrial machine will be needed, avoiding the requirement to perform a full surface response experiment design in the industrial machine.

As the models found for Ra in test and industrial machine are valid within the experimental region, it is required to generate forecasts within this experimental area. However, the dissimilarities in the velocities used in both machines make the experimental regions slightly different. So a common experimental region in the configurations of both machines must be defined in order to compare them.

Table 2 shows the levels for each factor in the simulated experiments, all of them being comprised within the common experimental region. It corresponds to tangential speed values between 20 and $25 \mathrm{~m} / \mathrm{min}$, and linear speed values between 30 and $35 \mathrm{~m} / \mathrm{min}$ were considered for the simulation experiments. 
Table 2. Variable levels used for the simulated experiments

\begin{tabular}{|c|c|c|c|c|}
\hline Factor & \multicolumn{4}{|c|}{ Levels } \\
\hline Gs & 15 & 20 & 25 & 30 \\
\hline $\mathrm{De}$ & 10 & 15 & 20 & \\
\hline $\mathrm{Pr}$ & 400 & 550 & 700 & \\
\hline $\mathrm{Vt}$ & 20 & 25 & & \\
\hline $\mathrm{Vl}$ & 30 & 35 & & \\
\hline
\end{tabular}

All possible combinations of the factors' levels $(4 \cdot 3 \cdot 3 \cdot 2 \cdot 2=144$ combinations $)$ were made to build the table of conditions for forecasting roughness. Using the models obtained in section 3.2 for the test and the industrial machines, values for response $\mathrm{Ra}$ were calculated. The prediction in all 144 combinations for the two machines can be seen in Figure 7.

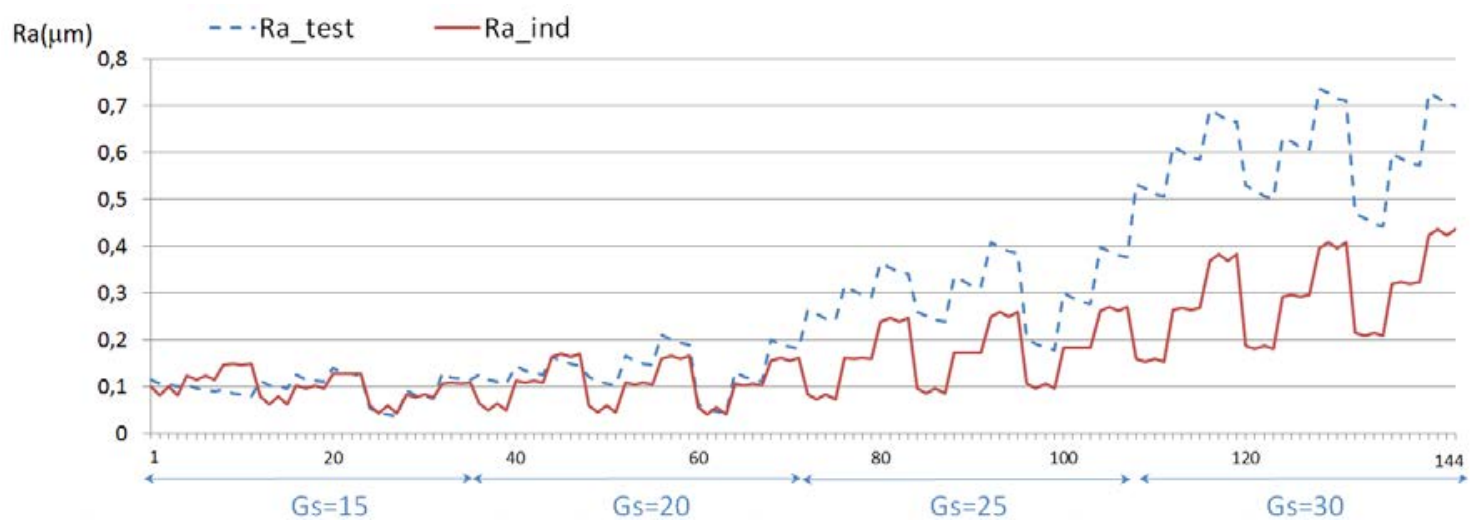

Figure 7. Comparison of predictions for Ra in the test machine (Ra_test) and in the industrial machine (Ra_ind)

Each abscissa value in Figure 7 corresponds to one of the 144 combinations of the factors studied, in standard order. For example, in combination 132 (where Gs $=30$ (FEPA), De = 15 (ISO6104), $\mathrm{Pr}=700 \mathrm{~N} / \mathrm{cm}^{2}, \mathrm{Vt}=25 \mathrm{~m} / \mathrm{min}$ and $\mathrm{Vl}=35 \mathrm{~min} / \mathrm{min}$ ), the prediction is $\mathrm{Ra}=0.41 \mu \mathrm{m}$ for the industrial machine and $\mathrm{Ra}=0.71 \mu \mathrm{m}$ for the test 
machine. It can be noticed that roughness of test machine is, in general, higher than the one of the industrial machine. The distance between both lines (Ra for test and industrial machines) depends, essentially, on the grain size (Gs), and increases with grain size, but nonlinearly. Grain size Gs is known to influence greatly surface roughness to be obtained, since the higher grain size the deeper grooves or furrows produced by honing operation are [12].

In order to create a model for the difference in average roughness between test and industrial machines (translation model), a new variable DifRa was defined (Equation 8):

$$
\text { DifRa }=R a_{\text {test machine }}-R a_{\text {industrial machine }} \quad \text { (Eq. 8) }
$$

Where $R a_{\text {industrial machine }}$ is the average roughness value from the industrial machine, and $R a_{\text {test machine }}$ is the average roughness value from the test machine

The model for DifRa is presented in Equation 9:

$$
\text { DifRa }=0.3442-0.04482 G s+0.001452 G s^{2} \quad \text { (Eq. 9) }
$$

The model has a coefficient of determination $R^{2}=95.52 \%$ and an adjusted $R^{2}=$ $95.43 \%$.

Although the regression equation was computed for all five variables, the only really active factor for the difference of roughness between machines is grain size (affecting in a quadratic manner). This result is completely coherent with the fact that Gs is by far the most important factor for $\mathrm{Ra}$ in both machines. This is an important result for the methodology: only grain size (Gs) has to be taken into account to "translate" results 
from the test machine to the industrial machine. Figure 8 shows this quadratic relationship.

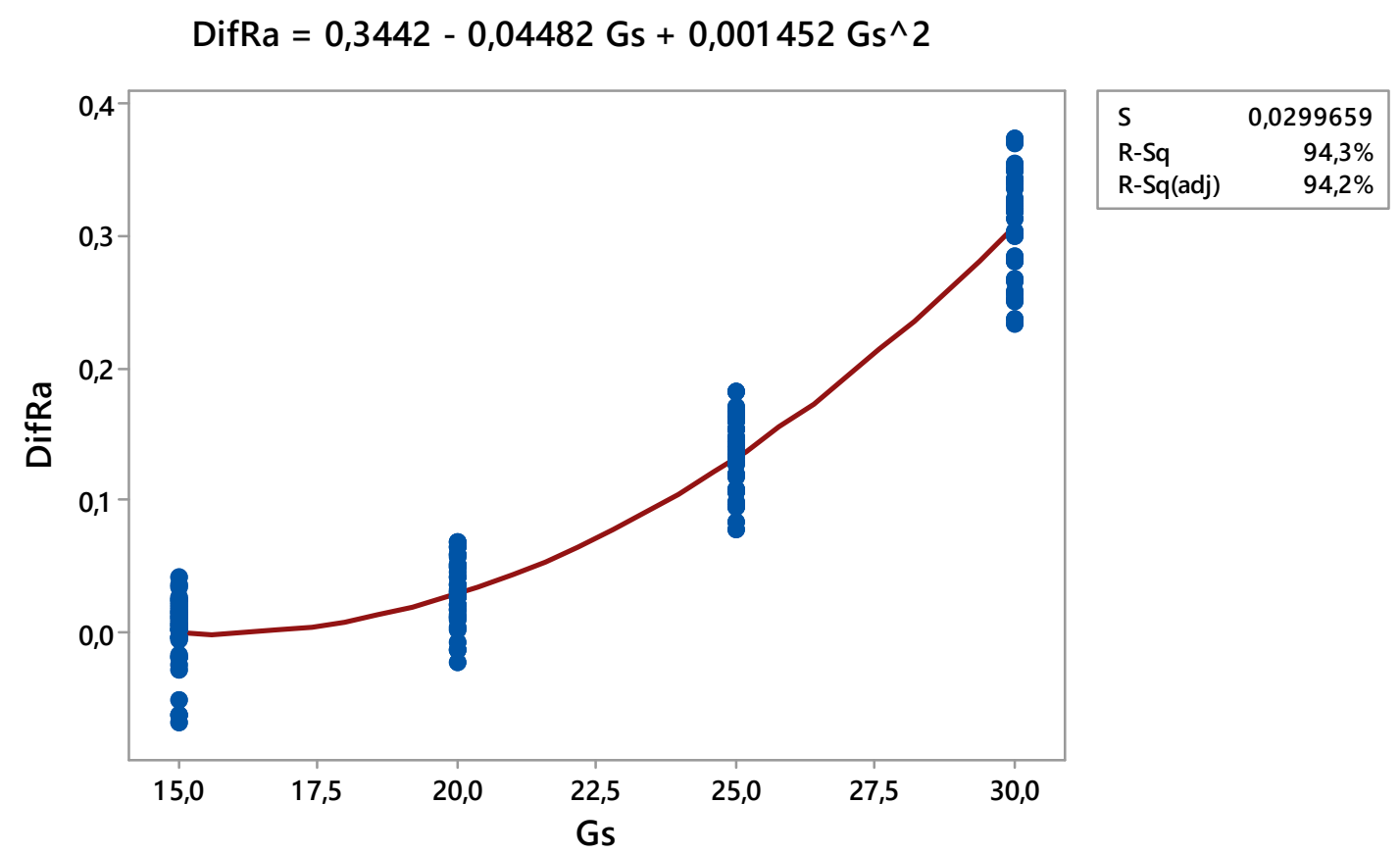

Figure 8. Differences in Ra vs Gs

The translation needed between machines can be deduced from Figure 8 and taking into account the obtained model for DifRa. When Gs $=15$, there is no translation, results are very similar for both machines. When $\mathrm{Gs}=20$, Ra in the industrial machine is lower, with a translation respect the test machine of approximately $0.03 \mu \mathrm{m}$. When Gs $=25$, the translation is $0.13 \mu \mathrm{m}$, and when $\mathrm{Gs}=30$, the translation is $0.31 \mu \mathrm{m}$.

To summarize, the suggested methodology for Ra in this case of finish honing processes is the following:

1. Conduct a test for each Gs value, keeping the rest of the variables constant, in both the test and the industrial machine. 
2. Compute the differences in roughness values between the industrial and the test machine for each grain size tested.

3. In future experimentations, the test machine can be used, with the only requirement of adding the obtained difference depending on the grain size for translating the value to the industrial machine.

\subsection{Models for other Roughtness parameters}

The methodology applied to Ra has been used with other measurements of roughness, such as Rz, Rk, Rvk and Rpk, in an attempt to check if the procedure works well for other magnitudes. The results obtained for $\mathrm{Rz}$ and $\mathrm{Rk}$ are detailed in subsections 3.3.1 and 3.3.2.

\subsubsection{Models for $\mathrm{Rz}$ and translation model for $\mathrm{Rz}$}

The fitted model for the test machine when using $\mathrm{Rz}$ as response is shown in Equation 10, whereas the model for the industrial machine is shown in Equation 11. It is important to notice how, in this case, the response had to be transformed using a logarithm, in order to get the best possible model for the data.

The model for the test machine is:

$$
\begin{aligned}
\log R z=-0.912 & -0.0280 G s-0.00771 D e+0.001282 P r+0.00367 V t-0.00462 V l \\
& +0.002926 G s^{2}
\end{aligned}
$$

(Eq. 10)

The model has a coefficient of determination $R^{2}=96.09 \%$ and an adjusted $R^{2}=$ $95.68 \%$. 
The model for the industrial machine is:

$$
\begin{aligned}
\log R z=4.161- & 0.2643 G s-0.1162 \mathrm{De}-0.001871 \mathrm{Pr}-0.0376 \mathrm{Vt}-0.00602 \mathrm{Vl}+0.004277 G s^{2} \\
& +0.004920 \mathrm{Gs} \cdot \mathrm{De}+0.000092 \mathrm{Gs} \cdot \mathrm{Pr}+0.000062 \mathrm{Pr} \cdot \mathrm{Vt}
\end{aligned}
$$

(Eq. 11)

The model has a coefficient of determination $R^{2}=88.42 \%$ and an adjusted $R^{2}=$ $86.41 \%$.

As it was done for Ra, the response for both the test machine and the industrial machine was simulated using the obtained models, in the common area of experimentation for both machines (Table 2). The result can be seen in Figure 9.

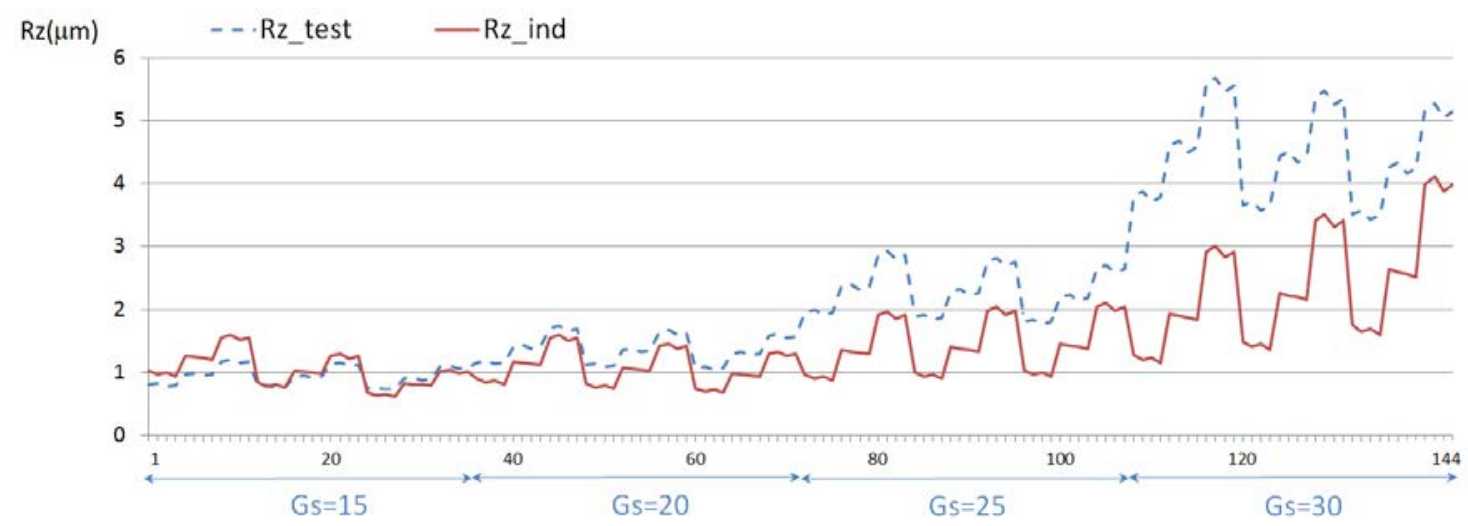

Figure 9. Comparison of predictions for $\mathrm{Rz}$ in the test machine (Rz_test) and in the industrial machine $\left(\mathrm{Rz} \_\right.$ind $)$

As before, only grain size defines the different translation areas. Equation 12 shows the model for the difference in $\mathrm{Rz}$ between the test machine and the industrial machine.

$$
\operatorname{DifRz}=1.662-0.2449 G s+0.008636 G s^{2}
$$

(Eq. 12) 
The difference of $\mathrm{Rz}$ between machines only depends on grain size in a quadratic manner, as shown in Figure 10.

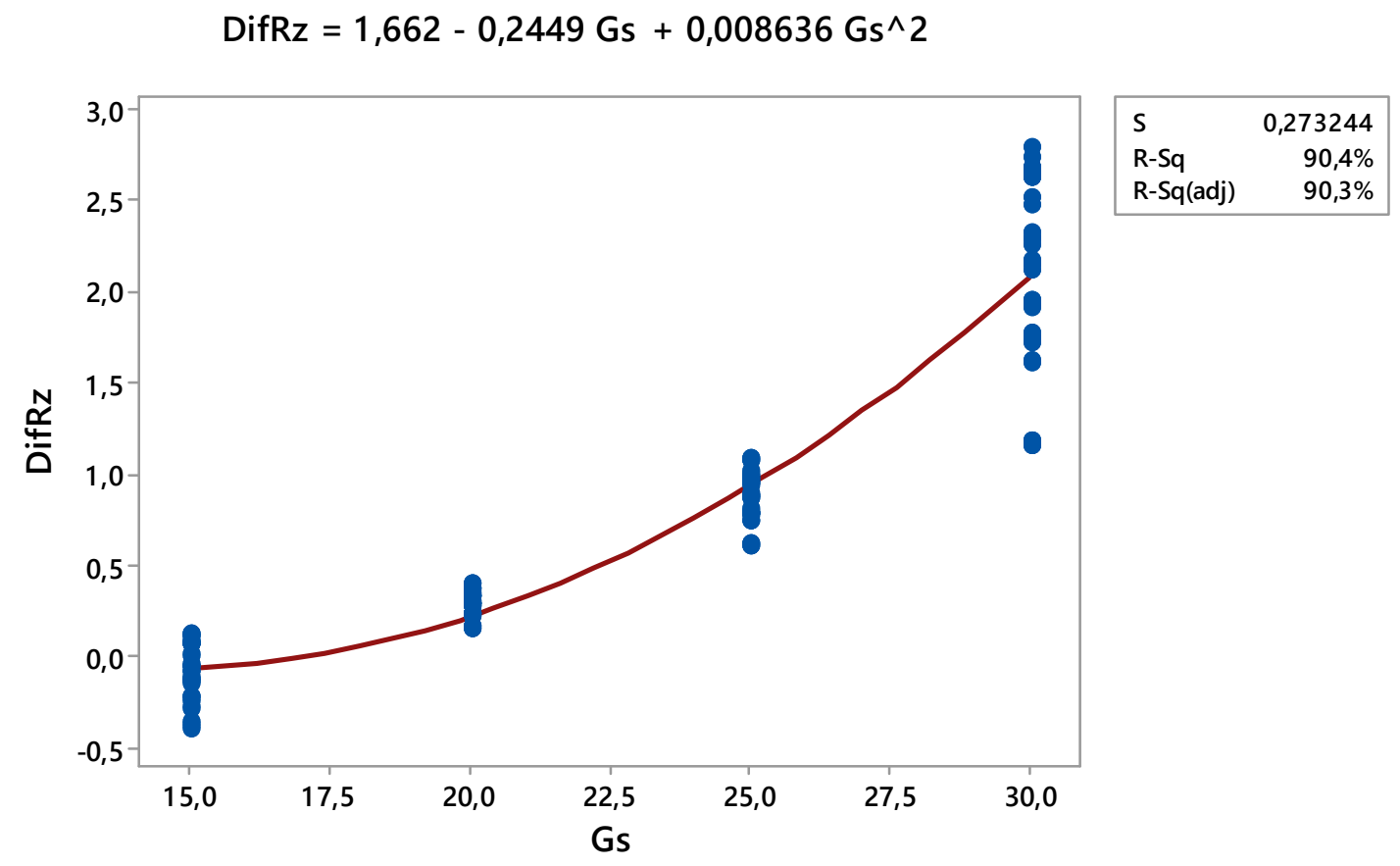

Figure 10. Differences in Rz vs Gs.

The result obtained for $\mathrm{Rz}$ is thus analogous to that obtained for $\mathrm{Ra}$. $\mathrm{Rz}$ in the industrial machine can be calculated from Rz in the test machine, just adding a translation constant that depends on the grain size.

\subsubsection{Models for $\mathbf{R k}$ and translation model for $\mathbf{R k}$}

The same methodology has been applied to parameters from the Rk family. The results for Rk are shown below, with identical results to those of Ra and Rz.

The fitted model for the test machine with Rk as response is shown in Equation 13. It has a coefficient of determination $R^{2}=99.2 \%$ and an adjusted $R^{2}=98.99 \%$. 


$$
\begin{gathered}
R k=3.427-0.3260 G s+0.0361 D e-0.00011 P r-0.02131 V t-0.02252 V l+0.008349 G s^{2} \\
+0.0278 D e^{2}-0.000003 P^{2}-0.000533 G s \cdot D e+0.000128 G s \cdot P r \\
-0.000454 G s \cdot V l+0,000102 \mathrm{De} \cdot \mathrm{Pr}+0,000734 \mathrm{Vt} \cdot V l
\end{gathered}
$$

(Eq. 13)

The fitted model for the industrial machine with $\mathrm{Rk}$ as response is shown in Equation 14. It has a coefficient of determination $R^{2}=87.64 \%$ and an adjusted $R^{2}=85.50 \%$.

$$
\begin{aligned}
\log R k=2,062 & -0,4178 G s+0,120 D e+0,000963 P r-0,0368 V t-0,00694 V l \\
& +0,00912 G s^{2}-0,00773 D e^{2}+0,00500 G s \cdot D e+0,000057 P r \cdot V t
\end{aligned}
$$

(Eq. 14)

The simulated responses from the test machine and the industrial machine can be seen in Figure 11.

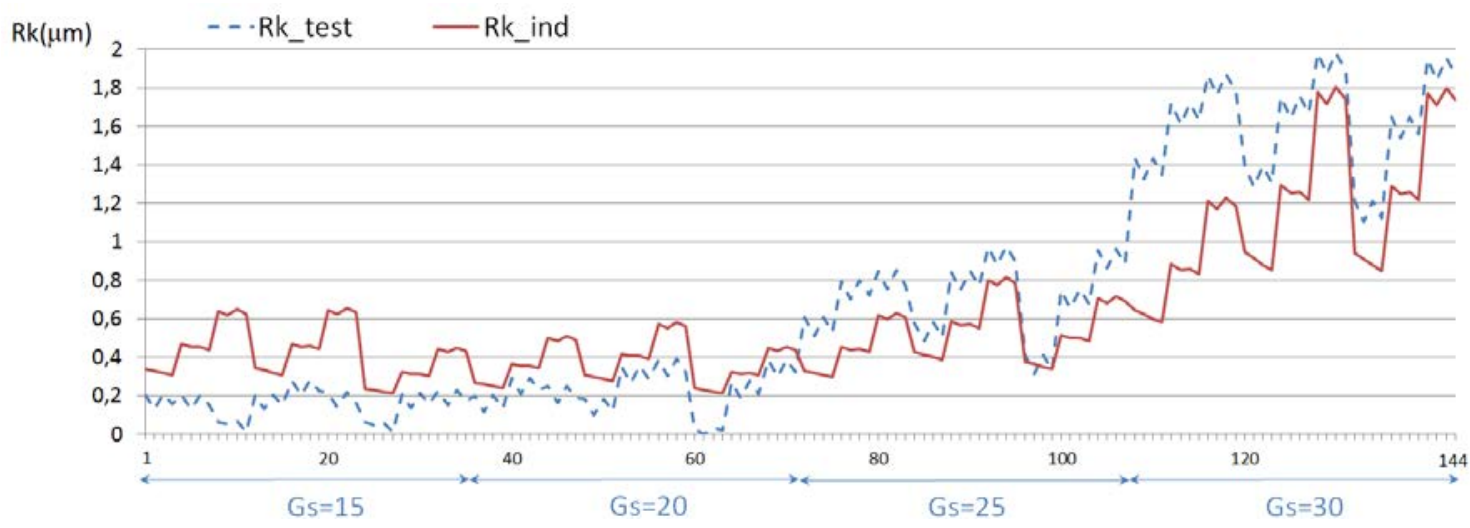

Figure 11. Comparison of predictions for $\mathrm{Rz}$ in the test machine (Rz_test) and in the industrial machine (Rz_ind)

Once again, only grain size defines the different translation areas. Equation 15 shows the model for the difference in $\mathrm{Rk}$ between the test machine and the industrial machine. 


$$
\operatorname{DifRk}=-0,397-0,0136 G s+0,001408 G s^{2}
$$

(Eq. 15)

This difference is graphically represented in Figure 12.

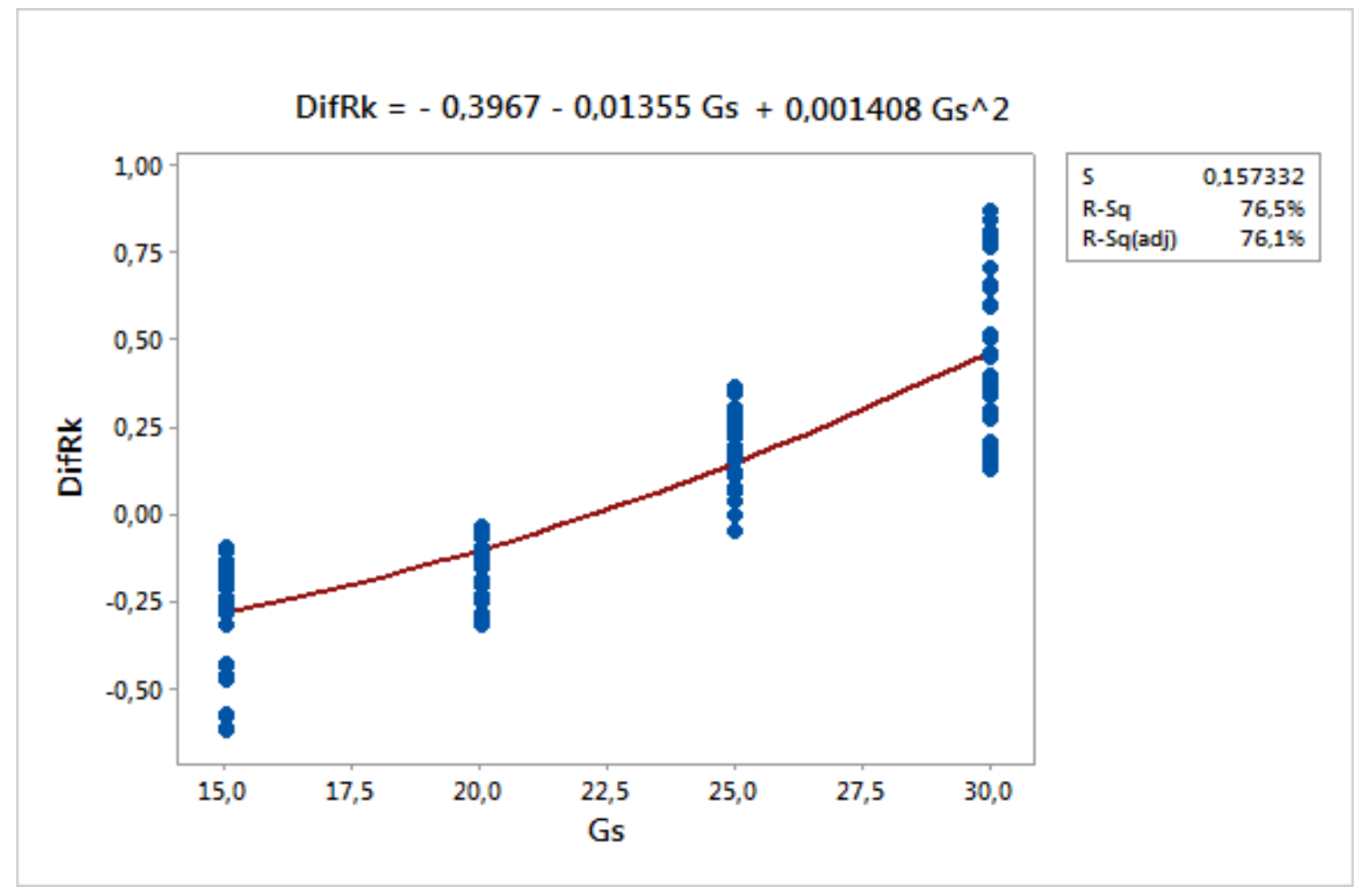

Figure 12. Differences in Rz vs Gs.

The result obtained for Rk is thus analogous to that obtained for Ra and in Rz. The same procedure has been applied to Rvk and Rpk, with the same successful results.

\subsection{Generalized translation methodology for roughness}

The different steps of the methodology that were presented in previous sections 3.1, 3.2 and 3.3 for predicting roughness values in an industrial machine from a test machine that can be schematized in Figure 13. This methodology is in fact general, and could be applied not only to finish honing processes, but to other mechanical processes that share 
the situation of having both a test machine and an industrial machine. The main requirement to use the methodology is that the model for the distance between values from test and industrial machines is simple enough to reduce number of tests to be conducted.

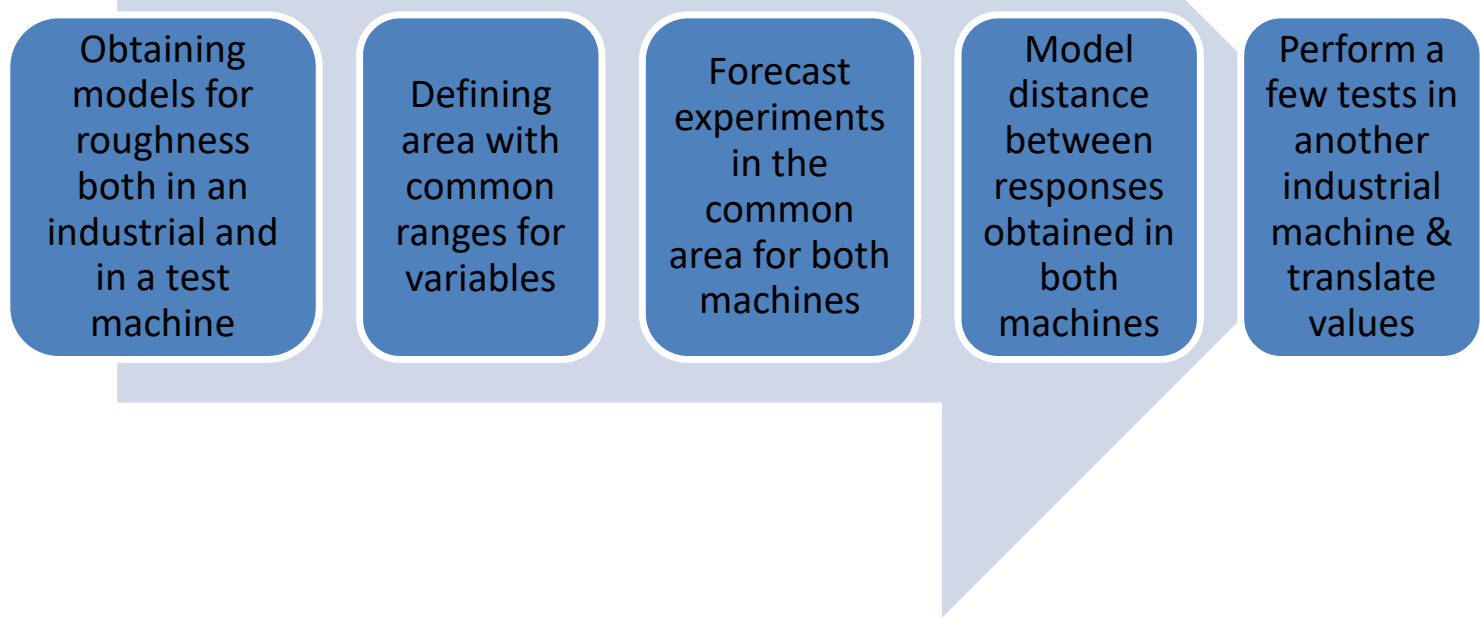

Figure 13. Methodology for obtaining roughness values in an industrial machine from a test machine

A description of the needed steps is provided below:

First, mathematical regression models are obtained from experimental data for a certain roughness parameter, both in a test and in an industrial machine. With the help of an analysis of relative importance of the factors, it might be possible to simplify the models obtaining reduced models, which are simpler than the complete ones.

Second, the experimental area where ranges of variables coincide for both machines is defined. Such area is called common area. 
Third, points are predicted in the common area using the models for the test and for the industrial machine.

Fourth, distance between results in the test and in the industrial machine (new response called Dif) is modeled. Depending on the number of factors affecting the distance, the number of different “translation areas” needed will differ.

Finally, several tests are to be performed in a different industrial honing machine to discover the translation values between the test machine and the industrial machine, depending on the factors affecting the variable Dif. These differences can then be used to translate results from the test to the industrial machine.

\section{Conclusions}

Main conclusions of the paper are presented next:

- A new methodology was created for predicting surface roughness to be obtained in honing industrial machines, from data obtained in test machines. This methodology allows translating results from a test machine to an industrial machine by only performing few tests in the industrial machine.

- Mathematical models were obtained for average roughness Ra in finish honing processes for both a test and an industrial machine. Main factor affecting roughness was grain size in both cases, followed by pressure.

- A new parameter DifRa was defined for modeling distance or difference between roughness values obtained in the test and in the industrial honing machine. In the range studied, DifRa depends mainly on grain size of abrasive. 
DifRa will allow translating roughness results from a test machine to an industrial machine without need to perform time-consuming honing tests.

- $\quad$ The devised procedure has been successfully applied to other roughness parameters such as Rz and Rk, and finally the methodology has been described in a general manner, making it ready to be tested in other variables and even other processes.

\section{Acknowledgements}

The authors are grateful to Dr. Joan Vivancos-Calvet for his valuable comments, as well as to Mr. Alejandro Domínguez-Fernández and Mr. Ramón Casado-López for their help with experimental tests. They also thank the Spanish Ministry of Economy and Competitiveness for project DPI2011-26300.

\section{References}

[1] Klocke F. Manufacturing Processes 2 - Grinding, Honing, Lapping. Springer; 2009.

[2] Schnitzler G. Honing: It’s no lapping matter! Manuf Eng 2000;125 (3):56-62.

[3] Yousfi M, Mezghani S, Demirci I, El Mansori M. Smoothness and plateauness contributions to the running-in friction and wear of stratified helical slide and plateau honed cylinder liners. Wear 2015;332:1238-47.

[4] Cabanettes F, Dimkovski Z, Rosén B-G. Roughness variations in cylinder liners induced by honing tools’ wear. Precis Eng 2015;41:40-6.

[5] Troglio AJ. Performance evaluation of multi-stone honing tool by experimental design methods. Proc. Int. Honing 2003 Conf., vol. MR03-232, 2003, p. 1-24.

[6] Bai YJ, Zhang LH, Ren CG. Experimental investigation on honing of small holes. Key Eng Mater 2007;329:303-8.

[7] Kanthababu M, Shunmugam MS, Singaperumal M. Identification of significant parameters and appropriate levels in honing of cylinder liners. Int J Mach Mach Mater 2009;5(1):80-96.

[8] Pawlus P, Cieslak T, Mathia T. The study of cylinder liner plateau honing process. Spec Issue 1st Int Conf Abras Process 2009;209:6078-86. 
[9] Buj-Corral I, Vivancos-Calvet J, Coba-Salcedo M. Modelling of surface finish and material removal rate in rough honing. Precis Eng 2014;38:100-8.

[10] Lawrence KD, Ramamoorthy B. Multi-surface topography targeted plateau honing for the processing of cylinder liner surfaces of automotive engines. Appl Surf Sci 2016;365:19-30.

[11] Vrac DS, Sidjanin LP, Kovac PP, Balos SS. The influence of honing process parameters on surface quality, productivity, cutting angle and coefficients of friction. Ind Lubr Tribol 2012;64:77-83.

[12] Bell SB, Maden H, Needham G. The influence of grit size and stone pressure on honing. Precis Eng 1981;3:47.

[13] Rao RV. Advanced Modeling and Optimization of Manufacturing Processes. London: Springer London; 2011.

[14] Buj-Corral I, Vivancos-Calvet J, Rodero-de-Lamo L, Marco-Almagro L. Comparison between Mathematical Models for Roughness Obtained in Test Machine and in Industrial Machine in Semifinish Honing Processes. Procedia Eng 2015;132:545-52.

[15] Buj-Corral I, Vivancos-Calvet J. Roughness variability in the honing process of steel cylinders with CBN metal bonded tools. Precis Eng 2011;35:289-93.

[16] Montgomery DC. Design and Analysis of Experiments. New York: John Wiley and Sons, Inc.; 2003.

[17] FEPA. 61/97 - FEPA standard for superabrasives grain sizes 1997.

[18] ISO. ISO 6104:2005 - Superabrasive products -- Rotating grinding tools with diamond or cubic boron nitride -- General survey, designation and multilingual nomenclature 2005.

[19] Minitab Inc. Minitab 172016.

[20] Chao Y-CE, Zhao Y, Kupper LL, Nylander-French LA. Quantifying the relative importance of predictors in multiple linear regression analyses for public health studies. J Occup Environ Hyg 2008;5:519-29. 